\title{
Reliability and validity of the Edinburgh Postnatal Depression Scale (EPDS) for detecting perinatal common mental disorders (PCMDs) among women in low-and lower-middle-income countries: a systematic review
}

\author{
Sumitra Devi Shrestha ${ }^{1,2,3^{*}}$, Rina Pradhan ${ }^{2,4}$, Thach D. Tran ${ }^{3}$, Rosa C. Gualano ${ }^{3}$ and Jane R. W. Fisher ${ }^{3}$
}

\begin{abstract}
Background: The Edinburgh Postnatal Depression Scale (EPDS), originally developed in Britain, is one of the most widely used screening instruments for assessing symptoms of the Perinatal Common Mental Disorders (PCMDs) of depression and anxiety. However, its potential to detect PCMDs in culturally diverse low- and lower-middle income countries (LALMICS) is unclear. This systematic review aimed to appraise formally validated local language versions of the EPDS from these resource-constrained settings.

Methods: Following the PRISMA protocol, we searched MEDLINE-OVID, CINAHL-Plus and PUBMED to identify studies reporting translation, cultural adaptation and formal validation of the EPDS to detect PCMDs among women in LALMICs. The quality of the studies meeting inclusion criteria was assessed using standard criteria and a new process-based criteria; which was developed specifically for this study.

Results: We identified 1281 records among which 16 met inclusion criteria; three further papers were identified by hand-searching reference lists. The publications reported findings from 12 LALMICs in 14 native languages. Most of these local language versions of the EPDS (LLV-EPDS) had lower precision for identifying true cases of PCMDs among women in the general perinatal population compared to the original English version. Only one study met all criteria for culturally sensitive translation, the others had not established the comprehensibility of the local version amongst representative groups of women in pre-testing. Many studies tested the LLV-EPDS only amongst convenience samples recruited at single health facilities. Diagnostic interviews for confirmation of mental disorders could have been influenced by the mental health professionals' lack of blinding to the initial screening results. Additionally, even when diagnostic-interviews were carried out in the local language, questions might not have been understood as most studies followed standard diagnostic protocol which had not been culturally adapted.

(Continued on next page)
\end{abstract}

\footnotetext{
* Correspondence: sumitra.d.shrestha@monash.edu

1 Ministry of Health and Population, Kathmandu, Nepal

${ }^{2}$ Life Line Nepal, Kathmandu, Nepal

Full list of author information is available at the end of the article
} 
(Continued from previous page)

Conclusions: Most of the LLV-EPDS from non-English speaking low- and middle-income-countries did not meet all criteria for formal validation of a screening instrument. Psychometric properties of LLV-EPDS could be enhanced by adopting the new process-based criteria for translation, adaptation and validation.

Keywords: Edinburgh postnatal depression scale (EPDS), Reliability, Validity, Cultural equivalence, Local language versions of the EPDS (LLV-EPDS), Perinatal common mental disorders (PCMDs), Low- and-lower-middle-income countries (LALMICS)

\section{Background}

There is growing recognition of mental health problems among women living in resource-constrained, World Bank defined low- and lower-middle-income countries (LALMICs) who are pregnant or have recently given birth. In order to address persistently high maternal and child morbidity and mortality and promote the survival, health and development of infants in these settings, national governments have expressed increasing interest in improving maternal mental health $[1,2]$. To optimize detection of Perinatal Common Mental Disorders (PCMDs) among women in primary healthcare, locally adapted and validated screening instruments are needed.

The Edinburgh Postnatal Depression Scale (EPDS) is one of the most widely used screening instruments for assessing symptoms of perinatal depression and anxiety $[3,4]$. It assesses emotional experiences over the past seven days using ten Likert-scale items (See Additional file 1). This self-reporting instrument was originally developed in the United Kingdom (U.K.) by Cox, Holden and Sagovsky in 1987 [5]. Its use has now extended far beyond the U.K. to other high-income English-speaking and non-English speaking countries, and increasingly to non-Anglophone LALMICs. The popularity of this brief instrument reflects the original British validation study [6], in which nine out of ten women who were diagnosed by a psychiatrist as being depressed after giving birth were correctly identified in a blinded comparison with scores above a cut-off on the EPDS. The psychometric properties of the EPDS in primary health care were: $86 \%$ sensitivity (correctly identifying true cases), $78 \%$ specificity (correctly identifying people without the condition) and $73 \%$ positive predictive value (proportion of respondents scoring positive in the test who had a mental disorder diagnosed by clinical interview) [6].

To improve early detection and treatment of PCMDs, the local language versions of the EPDS (LLV-EPDS) needs to accurately identify people with a PCMDs. However, it has been found that the LLV-EPDS had relatively lower discriminant validity for correctly identifying cases of PCMDs [3, 4] than the original English version [6]. Many reasons have been proposed for why LLV-EPDS did not perform well. These include lack of local cultural sensitivity [3, 7-9] due to compromises made during translation and adaptation process [10] and recruitment of participants who did not represent general perinatal populations [4]. Finally, the questions asked during diagnostic interviews (the standard comparator) might not have been meaningful or comprehensible in these local settings. Development of a LLV-EPDS with optimal psychometric properties is fundamental for identification of perinatal mental disorders, for assisting nations to assess the overall burden of PCMDs [11] and enabling aggregation of global prevalence data [10]. However, there is no internationally approved, standard technique for translation and validation of the English EPDS into a nonEnglish local language version appropriate for use in resource-constrained countries.

There are three systematic reviews on the validity of non-English versions of the EPDS. Two of these studies were focused on publications from high and middleincome countries, and included few studies from lowincome countries $[3,4]$, while the other review included only data from African countries [12]. There is no systematic review specific to low-and lower-middle-income countries (LALMICs) [13]. The objectives of this review were: (1) to appraise systematically the formally validated LLV-EPDS from LALMICs, and (2) to establish potentially modifiable reasons for their lower validity by using new specific process-based criteria.

\section{Methods \\ Search strategy}

We used the Preferred Reporting Items for Systematic reviews and Meta-Analyses (PRISMA) protocol for identifying, screening and eligibility of studies [14] (Additional file 2). Three indexed electronic international databases (MEDLINE-OVID, CINAHL-Plus and PUBMED) were searched up to 20 April 2015, using the strategy described in Additional file 3.

\section{Inclusion criteria}

There were four inclusion criteria: studies on translation and/or cultural adaptation and/or validation of the EPDS; that enrolled women who were pregnant and/or had recently given birth; which were conducted in World Bank defined LALMICs, and with reports published in English language, peer-reviewed journals. 


\section{Selection of studies}

In addition to implementing the search strategy, the reference lists of articles meeting inclusion criteria were searched to identify any studies that had not been found. In order to obtain copies of studies published in nonindexed or local journals, we corresponded with authors via email, if we did not receive a response we wrote to the editor of the journal. By learning the journal was no more published, we sought a copy of the publications through interlibrary loan.

\section{Quality assessment}

We used two approaches to assess the methodological quality of the selected publications. First, overall quality was assessed using the criteria recommended by Mirza and Jenkins [15]. As recommended by Fisher et al. [1] we added a criterion about whether approval from a formally constituted ethics committee had been obtained. Thus, the 9 criteria were: (1) clear study aim; (2) sufficient sample size or justification; (3) representativeness of the sample or justification; (4) explicit inclusion and exclusion criteria; (5) response rate and explanation of losses; (6) clear description of data; (7) appropriate statistical analyses; (8) ethics approval; (9) and obtained informed consent. One point was given for meeting each of these points (1for Yes and 0 for No), to yield a maximum total possible score of 9 .

The Mirza \& Jenkins [15] and Fisher et al. [1] assessment scheme did not include specific criteria for assessing quality of a screening instrument like the EPDS. We assessed quality of the translation, cultural adaptation and local validation of the LLV-EPDS by developing a new set of process-based criteria (shown in Fig. 1, and defined in Additional file 4). We derived 33 criteria from diverse sources: which were recommended points for translation of other psychometric instruments $[10,16]$ and self-reporting questionnaires (SRQ) [17], measures used and suggested for translation and validation of the EPDS by earlier studies [3, 7, 11, 18-22]. Additionally, we incorporated some criteria based on our experience in international public health.

\section{Collection of data and analysis}

We extracted data from selected studies using dataextraction format (Table 1).

Adherence to the 33 process-based criteria were organised into two sections: Culturally Sensitive Translation and Empirical Validation using three response options: Yes/Not mentioned/Not needed (Tables 3 and 4). In line to aim of this study, data (evidence) about adherence to the process-based criteria summarised as narratives by three key aspects of LLV-EPDS development process: Culturally Sensitive Translation, Empirical Validation and Psychometric Properties (meta-analysis was not done as was beyond our study objectives). The data on processbased criteria and methodological quality were extracted by the first author and then rechecked by other authors; differences were resolved by consensus.

\section{Results}

In total 1281 records were identified using the search strategy, after removal of duplicates and studies which did not meet inclusion criteria, including six articles published in languages other than English (French, Lithuanian, Polish, Turkish, Hebrew and German), but not conducted in LALMICs,19 studies, all quantitative, remained (Fig. 2 and Table 1).

Methodological assessment of these 19 selected publications indicated that five studies had enrolled sufficient participants to achieve adequate power. Overall, all had a clearly stated aim, 16 studies used clearly defined criteria for selection of the participants. Nine reported representativeness of samples with justification, 12 provided the recruitment rate, 14 publications included a synopsis of participants' characteristics, 14 acquired ethical approvals, three studies described how participants' consent had been acquired, but the remaining studies did not. Almost all studies had used appropriate statistical analyses for deriving psychometric properties (Table 2). All studies were included in this review despite of their modest quality. Since, this study aimed to identify reasons behind formally validated LLV-EPDS having lower validity than the original English version.

Of these 19 publications, two studies described translations of the original English EPDS into local languages, one entirely on the topic and another dealt briefly [19, 23]; 13 studies discussed both translation and psychometric properties of the LLV-EPDS; and four studies [24-27] focused on establishing psychometric properties for LLV-EPDS that were already translated. In total 17 studies described psychometric properties of the LLV-EPDS by recruiting a total of 4029 women (sample sizes ranged from 100 to 601) from 12 LALMICs [24-40].

\section{Culturally Sensitive Translation}

We found 15 publications reporting translations and adaptation of 14 LLV-EPDS from 12 countries; there were two studies from each of three countries. India $[28,29]$ and Nigeria $[30,31]$ each had two native language versions of the EPDS. Both studies from Ethiopia [33, 35] were related to the development of an Amharic version of the EPDS.

In these 15 studies there was variable application of the six key steps we proposed for the culturally sensitive translation of the EPDS: forward translation, backward translation, resolution of difficulties and differences in translations by committee approach, pretesting, amendments and test of conceptual and operational equivalence 


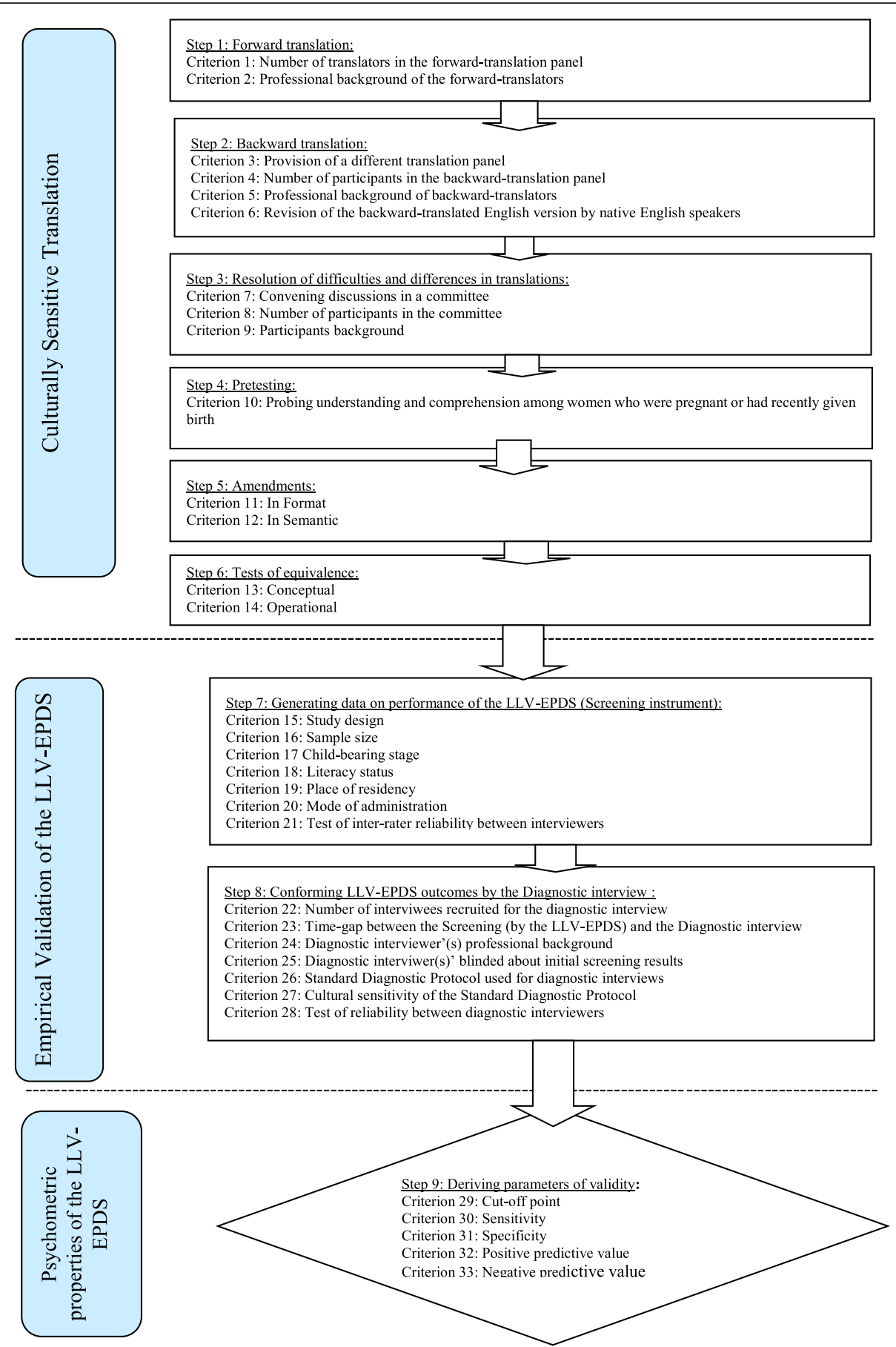

Fig. 1 Development of Process-based review criteria for assessing formally validated local language versions of the EPDS (LLV-EPDS) in low- and lower-middle-income countries

(Table 3). Seven studies described the translation process and reported forward and backward translation by native speakers who were fluent in English as well. There was a predominance of health professionals in translation panels, which typically had 1-4 members. Two forwardtranslation panels $[19,32]$ had included professional translators. In eight studies there were separate panels
[19, 24, 29-34] for forward and backward translations. Only one study reported review of the back-translated version by native English speakers [19].

Nine studies resolved differences in translation by convening a large group discussion; however in most of these studies (8/9), such discussions were held amongst diverse health professionals and in two studies professional 
Table 1 Studies included for the systematic review on reliability and validity of the Edinburgh Postnatal Depression Scale in low- and lower-middle-income countries $\begin{array}{ll}\text { 3. Patel et al., } & \text { Hospital in the Northern } \\ \text { (2002) [28] } & \text { town of Goa (Western }\end{array}$ State), India.

$\begin{array}{ll}\text { 4. Uwakwe } & \text { University teaching } \\ \text { (2003) [30] } & \begin{array}{l}\text { hospital, located in } \\ \text { the Eastern State, }\end{array}\end{array}$

Nigeria.

5. Fisher et al., (2004) [23]

Maternal and Child and Family Planning Centre, located in the Ho Chi Minh city, Viet Nam.

6. Rahman et Rural sub-district of

al., (2005) [39] Rawalpindi, Pakistan.

7. Adewuya et 5 Health centres, al.. (2006) [31] located in a semi-urban

Study type Sample and recruitment

Outcome Variable

Cross-

sectional

- 132/149 postnatal
women

women

- Recruited from the

maternity wards of

respective hospitals

( $\geq 2$ day's post-delivery)

and followed up after

4 weeks.

Cross- $\quad \cdot 100$ postnatal women

sectional (2-3 months)

- Recruited from the

post-natal/child

immunization clinic. It

recruited 40 non-child

bearing women as

$\&$ their friends).

Prospective • 270/297 pregnant

women ( $\geq 30$ week)

- Recruited from the

antenatal clinics, and

then followed 6-8

weeks after delivery.

Cross- $\quad \cdot$ 225/292 postnatal

sectional women

- Recruited from the

maternity ward $(\geq 7$

day's post-delivery) \&

then followed in the

first postnatal clinic

visit.

Cross- $\quad$ Cross-sectional, 506

sectional postnatal women (6-8

weeks)

- Recruited from the

infant health clinics or

those came for medical

review

Cross- $\quad \cdot 541 / 570$ postnatal

sectional women (10 to 12

weeks)

- Recruited from

community

Cross- 182 pregnant women

sectional ( $\geq 32$ weeks)

prevalence

PCMDs prevalence

\section{Psychometric properties}

Outcome Measured

Cut-off point; Sensitivity (Se)

Specificity (Sp); Positive

predictive value (PPV);

Negative predictive value

(NPV)

Psychometric properties

Cut-off point; Sensitivity (Se)

Specificity (Sp); Positive

predictive value (PPV):

Negative predictive value (NPV)

Psychometric propertiesPCMDs - Cut-off point; Sensitivity

(Se); Specificity (Sp)

- PCMDs prevalence

Psychometric properties

Cut-off point; Sensitivity (Se)

Specificity (Sp); Positive

predictive value (PPV):

Negative predictive value

(NPV)

PCMDs prevalence

Psychometric properties

Cut-off point; Sensitivity (Se):

Specificity (Sp); Positive predictive

value (PPV)

Psychometric properties

Cut-off point; Sensitivity (Se); sychometric properties

Nepalese version

- Cut-off point: 12/13

Se. 68.4

PPV: 65

- NPV: 94.6

Nepalese version

- Cut-off point: 12/13

- Se: 100

- Sp: 92.6

- NPV: 41.6

Konkani version:

- Cut-off point: 11/12

- Se: $92 \cdot$ Sp: 85

Igbo version (Eastern)

- Cut-off point: 8/9

- Se: $75 \cdot$ Sp: 97

- PPV: $75 \cdot$ NPV: 97

Reason included for this Review Earlier Vietnamese version-EPDS (developed by Small et al., (1999) [43] for women living in Australia) was revised before assessment

of prevalence

Urdu version

- Cut-off point: $9 / 10^{\mathrm{a}}$

- Se: 81.5

- Sp: 73.5

- PPV: 52.6

Specificity (Sp); Positive predictive value (PPV):
Yoruba version (Western)

- Cut-off point: 9/10

- Se: 86.7 
Table 1 Studies included for the systematic review on reliability and validity of the Edinburgh Postnatal Depression Scale in low- and lower-middle-income countries (Continued)

\begin{tabular}{|c|c|c|c|c|c|c|}
\hline & $\begin{array}{l}\text { town of the Western } \\
\text { Nigeria. }\end{array}$ & & $\begin{array}{l}\text { - Recruited from } \\
\text { antenatal clinics. }\end{array}$ & & $\begin{array}{l}\text { Negative predictive value } \\
\text { (NPV) }\end{array}$ & $\begin{array}{l}\text { - Sp: } 91.5 \\
\text { - PPV: } 68.4 \\
\text { - NPV: } 97\end{array}$ \\
\hline $\begin{array}{l}\text { 8. Pollock et al., } \\
\text { (2006) [32] }\end{array}$ & $\begin{array}{l}\text { Central Psychiatric } \\
\text { Hospital, Mental Health } \\
\text { and Necrology Centre, } \\
\text { and } 3 \text { Primary Health } \\
\text { Care Centres (PHCCs), } \\
\text { located in the } \\
\text { Ulaanbaatar (capital), } \\
\text { Mongolia. }\end{array}$ & $\begin{array}{l}\text { Cross- } \\
\text { sectional }\end{array}$ & $\begin{array}{l}\text {-94/100 women (in } \\
\text { reproductive age) } \\
\text { - Recruited from } \\
\text { psychiatric units ( } 55 \text { ) } \\
\text { - Rest from PHCCs } \\
\text { immunization clinics }\end{array}$ & Psychometric properties & $\begin{array}{l}\text { Cut-off point; Sensitivity (Se); } \\
\text { Specificity (Sp); Positive } \\
\text { predictive value (PPV); } \\
\text { Negative predictive value } \\
\text { (NPV) }\end{array}$ & $\begin{array}{l}\text { Mongolian version } \\
\text { - Cut-off point: } 12 / 13 \\
\text { - Se: } 80.9 \\
\text { - Sp: } 61.7 \\
\text { - PPV: } 67.9 \\
\text { - NPV: } 76.3\end{array}$ \\
\hline $\begin{array}{l}\text { 9. Gausia et al., } \\
\text { (2007) [19] }\end{array}$ & $\begin{array}{l}\text { Social and Behavioural } \\
\text { Sciences Unit (SBSU) } \\
\text { and Hospital, located } \\
\text { in the Dhaka (capital), } \\
\text { Bangladesh. }\end{array}$ & $\begin{array}{l}\text { Cross- } \\
\text { sectional }\end{array}$ & $\begin{array}{l}\text { - } 10 \text { female employee } \\
\text { from SBSU } \\
\cdot 11 \text { mothers (baby } \leq \\
1 \text { year) attending } \\
\text { immunization clinic } \\
.4 \text { women whose } \\
\text { infants were admitted } \\
\text { to the hospital }\end{array}$ & $\begin{array}{l}\text { Cultural and operational } \\
\text { equivalence of Bangla version } \\
\text { EPDS }\end{array}$ & $\begin{array}{l}\text { Correlation between Bangla } \\
\text { and original English version } \\
\text { Correlation between self- } \\
\text { report and interview } \\
\text { administration }\end{array}$ & $\begin{array}{l}\text { - Bangla and original English } \\
\text { version }(0.981 ; p<0.01) \\
\text { - Self-report and interview } \\
((0.752 ; p=0.01)\end{array}$ \\
\hline $\begin{array}{l}\text { 10. Gausia et al., } \\
\text { (2007) [25] }\end{array}$ & $\begin{array}{l}\text { Hospital, located in the } \\
\text { Dhaka (capital), } \\
\text { Bangladesh. }\end{array}$ & $\begin{array}{l}\text { Cross- } \\
\text { sectional }\end{array}$ & $\begin{array}{l}\cdot 100 / 126 \text { postnatal } \\
\text { women ( } 6 \text {-8 weeks) } \\
\text { • Recruited from child } \\
\text { immunization clinic. }\end{array}$ & Psychometric properties & $\begin{array}{l}\text { Cut-off point; Sensitivity (Se); } \\
\text { Specificity (Sp); Positive } \\
\text { predictive value (PPV); } \\
\text { Negative predictive value } \\
\text { (NPV) }\end{array}$ & $\begin{array}{l}\text { Bangla version } \\
\text { - Cut-off point: } 9 / 10 \\
\text { - Se: } 88.9 \\
\text { - Sp: } 86.8 \\
\text { - PPV: } 40 \\
\text { - NPV: } 98.6\end{array}$ \\
\hline $\begin{array}{l}\text { 11. Rowel et al., } \\
\text { (2008) [37] }\end{array}$ & $\begin{array}{l}\text { Field polyclinics in } \\
\text { Kolonnawa, Western } \\
\text { part of the Colombo } \\
\text { (capital), Sri Lanka. }\end{array}$ & $\begin{array}{l}\text { Cross- } \\
\text { sectional }\end{array}$ & $\begin{array}{l}\text { - } 465 \text { perinatal women } \\
\text { recruited: } \\
\text { - } 265 \text { pregnant ( } \geq 34 \\
\text { weeks) attending } \\
\text { antenatal clinics. } \\
\text { - } 204 \text { postpartum } \\
\text { women ( } \geq 6 \text { weeks) } \\
\text { attending family } \\
\text { planning or child } \\
\text { wellbeing clinics. }\end{array}$ & Psychometric properties & $\begin{array}{l}\text { Cut-off point; Sensitivity (Se); } \\
\text { Specificity (Sp) }\end{array}$ & $\begin{array}{l}\text { Sinhalese version } \\
\text { - Cut-off point: } 8 / 9 \\
\text { Pregnant } \\
\text { - Se: } 90.7 \\
\text { - Sp: } 86.8 \\
\text { Postnatal } \\
\text { - Se: } 89.9 \\
\text { - Sp: } 78.9\end{array}$ \\
\hline $\begin{array}{l}\text { 12. Hanlon et } \\
\text { al., (2008) [33] }\end{array}$ & $\begin{array}{l}\text { Butajra (rural region) } \\
\text { located } 130 \mathrm{~km} \text { South } \\
\text { of the Addis Abba } \\
\text { (capital), Ethiopia. }\end{array}$ & $\begin{array}{l}\text { Cross- } \\
\text { sectional }\end{array}$ & $\begin{array}{l}\cdot 101 \text { postnatal women } \\
\text { (median } 5 \text { Months) } \\
\text { - Recruited from the } \\
\text { community. }\end{array}$ & Psychometric properties & $\begin{array}{l}\text { Cut-off point; Sensitivity (Se); } \\
\text { Specificity (Sp) }\end{array}$ & $\begin{array}{l}\text { Amharic version } \\
\text { 1. Cut-off point: } 5 / 6 \\
\text { - Sp: } 76.5 \\
\text { - Se: } 36.1\end{array}$ \\
\hline $\begin{array}{l}\text { 13. Weobong } \\
\text { et al., (2009) } \\
\text { [38] }\end{array}$ & $\begin{array}{l}\text { Brong-Ahafo region } \\
\text { (South part), Ghana. }\end{array}$ & $\begin{array}{l}\text { Cross- } \\
\text { sectional }\end{array}$ & $\begin{array}{l}\text { - } 160 \text { pregnant women } \\
\text { (5-11 week) } \\
\text { - Identified from the } \\
\text { database of } 1 / 6 \text { districts } \\
\text { where vitamin A trial } \\
\text { initiated. }\end{array}$ & Psychometric properties & $\begin{array}{l}\text { Cut-off point; Sensitivity (Se); } \\
\text { Specificity (Sp); Positive } \\
\text { predictive value (PPV); } \\
\text { Negative predictive value } \\
\text { (NPV) }\end{array}$ & $\begin{array}{l}\text { Twi version } \\
\text { - Cut-off point: 10/11 } \\
\text { - Se: } 78 \\
\text { - Sp: } 73 \\
\text { - PPV: } 22 \\
\text { - NPV: } 97\end{array}$ \\
\hline
\end{tabular}


Table 1 Studies included for the systematic review on reliability and validity of the Edinburgh Postnatal Depression Scale in low- and lower-middle-income countries (Continued)

\begin{tabular}{|c|c|c|c|c|c|c|}
\hline $\begin{array}{l}\text { 14. Tesfaye et } \\
\text { al., (2010) [35] }\end{array}$ & $\begin{array}{l}2 \text { Primary Health Care Centres, } \\
\text { located in peri-urban area } \\
\text { of the Addis Ababa } \\
\text { (capital), Nigeria. }\end{array}$ & $\begin{array}{l}\text { Cross- } \\
\text { sectional }\end{array}$ & $\begin{array}{l}\text { - 100/102 postnatal } \\
\text { women (6 to } 14 \text { weeks) } \\
\text { - Recruited from child } \\
\text { immunization and } \\
\text { postnatal clinics. }\end{array}$ & Psychometric properties & $\begin{array}{l}\text { Cut-off point; Sensitivity (Se); } \\
\text { Specificity (Sp); Positive } \\
\text { predictive value (PPV); } \\
\text { Negative predictive value } \\
\text { (NPV) }\end{array}$ & $\begin{array}{l}\text { Amharic version } \\
\text { - Cut-off point: } 6 / 7 \\
\text { - Se: } 78.9 \\
\text { - Sp: } 75.3 \\
\text { - PPV: } 42.9 \\
\text { - NPV: } 93.8\end{array}$ \\
\hline $\begin{array}{l}\text { 15. Chibanda et } \\
\text { al., (2010) [36] }\end{array}$ & $\begin{array}{l}2 \text { Primary Health Care Centres } \\
\text { located in peri-urban area of } \\
\text { the Harare (capital), Zimbabwe. }\end{array}$ & $\begin{array}{l}\text { Cross- }^{-} \\
\text {sectional }\end{array}$ & $\begin{array}{l}\text {-210/223 postnatal } \\
\text { women (6-7 weeks) } \\
\text { - Identified by computer } \\
\text { generated randomization } \\
\text { of clinic review cards, } \\
\text { - Recruited from the } 2 \\
\text { primary health care } \\
\text { centres }\end{array}$ & Psychometric properties & $\begin{array}{l}\text { Cut-off point; Sensitivity (Se); } \\
\text { Specificity (Sp); Positive } \\
\text { predictive value (PPV); } \\
\text { Negative predictive value } \\
\text { (NPV) }\end{array}$ & $\begin{array}{l}\text { Shona version } \\
\text { - Cut-off point: 10/11 } \\
\text { - Se: } 88 \\
\text { - Sp: } 87 \\
\text { - PPV: 74NPV: } 94\end{array}$ \\
\hline $\begin{array}{l}\text { 16. Fernandes } \\
\text { et al. (2010) [29] }\end{array}$ & $\begin{array}{l}\text { Missionary hospital located in } \\
\text { rural part of Karnataka State } \\
\text { (South), India. }\end{array}$ & $\begin{array}{l}\text { Cross- } \\
\text { sectional }\end{array}$ & $\begin{array}{l}\text { - 194/196 pregnant } \\
\text { (32 - } 38 \text { weeks) women } \\
\text { - Recruited from the } \\
\text { antenatal clinic. }\end{array}$ & Psychometric properties & $\begin{array}{l}\text { Cut-off point; Sensitivity (Se); } \\
\text { Specificity (Sp); Positive } \\
\text { predictive value (PPV); } \\
\text { Negative predictive value } \\
\text { (NPV) }\end{array}$ & $\begin{array}{l}\text { Kannada version } \\
\text { - Cut-off point: 12/13 } \\
\text { - Se: } 100 \\
\text { - Sp: } 84.9 \\
\text { - PPV: } 52 \\
\text { - NPV: } 99\end{array}$ \\
\hline $\begin{array}{l}\text { 17. Tran et al., } \\
\text { (2011) [27] }\end{array}$ & $\begin{array}{l}\text { Randomly selected Commune } \\
\text { Health Centres (CMCs) from } \\
\text { the Hanoi (capital) and Ha } \\
\text { Nam province, Vietnam. }\end{array}$ & $\begin{array}{l}\text { Cross- }^{-} \\
\text {sectional }\end{array}$ & $\begin{array}{l}\text { - } 364 / 392 \text { perinatal } \\
\text { women } \\
\text { - } 199 \text { were } \geq 28 \text { weeks } \\
\text { pregnant } \\
\text { - Rest were 4-6 weeks } \\
\text { postpartum) } \\
\text { - Mostly were recruited } \\
\text { from the CMCs and in } \\
\text { Ha Nam province, also } \\
\text { house visit for some. }\end{array}$ & Psychometric properties & $\begin{array}{l}\text { Cut-off point; Sensitivity (Se); } \\
\text { Specificity (Sp); Positive } \\
\text { predictive value (PPV); } \\
\text { Negative predictive value } \\
\text { (NPV) }\end{array}$ & $\begin{array}{l}\text { Vietnamese version } \\
\text { - Cut-off point: } 3 / 4^{\mathrm{a}} \\
\text { - Se: } 69.9 \\
\text { - Sp: } 72.9 \\
\text { - PPV: } 69.7 \\
\text { - NPV: } 72\end{array}$ \\
\hline $\begin{array}{l}\text { 18. Husain et } \\
\text { al., (2013) [26] }\end{array}$ & $\begin{array}{l}\text { An urban slum in the Karachi } \\
\text { (capital), Pakistan. }\end{array}$ & $\begin{array}{l}\text { Cross- } \\
\text { sectional }\end{array}$ & $\begin{array}{l}\text { - } 601 / 664 \text { postnatal } \\
\text { women (0-36 months) } \\
\text { - Recruited from the slum. }\end{array}$ & Psychometric properties & $\begin{array}{l}\text { Cut-off point; Sensitivity (Se); } \\
\text { Specificity (Sp); Positive } \\
\text { predictive value (PPV); } \\
\text { Negative predictive value } \\
\text { (NPV) }\end{array}$ & $\begin{array}{l}\text { Urdu version } \\
\text { - Cut-off point: } 13 / 14^{a} \\
\text { - Se: } 79 \\
\text { - Sp: } 74 \\
\text { - PPV: } 82 \\
\text { - NPV: } 70\end{array}$ \\
\hline $\begin{array}{l}\text { 19. Stewart et } \\
\text { al., (2013) [34] }\end{array}$ & $\begin{array}{l}\text { District hospital, Mangochi, } \\
\text { (Southern township), Malawi. }\end{array}$ & $\begin{array}{l}\text { Cross- } \\
\text { sectional }\end{array}$ & $\begin{array}{l}\text { - } 224 \text { pregnant women } \\
\text { (2nd trimester) } \\
\text { - Recruited from } \\
\text { antenatal clinic. }\end{array}$ & Psychometric properties & $\begin{array}{l}\text { Cut-off point; Sensitivity (Se); } \\
\text { Specificity (Sp); Positive } \\
\text { predictive value (PPV); } \\
\text { Negative predictive value } \\
\text { (NPV) }\end{array}$ & $\begin{array}{l}\text { Chichewa version } \\
\text { - Cut-off point: } 4 / 5^{a} \\
\text { - Se: } 68.7 \\
\text { - Sp: } 88.2 \\
\text { - PPV: } 35.8 \\
\text { - NPV: } 97.4\end{array}$ \\
\hline
\end{tabular}




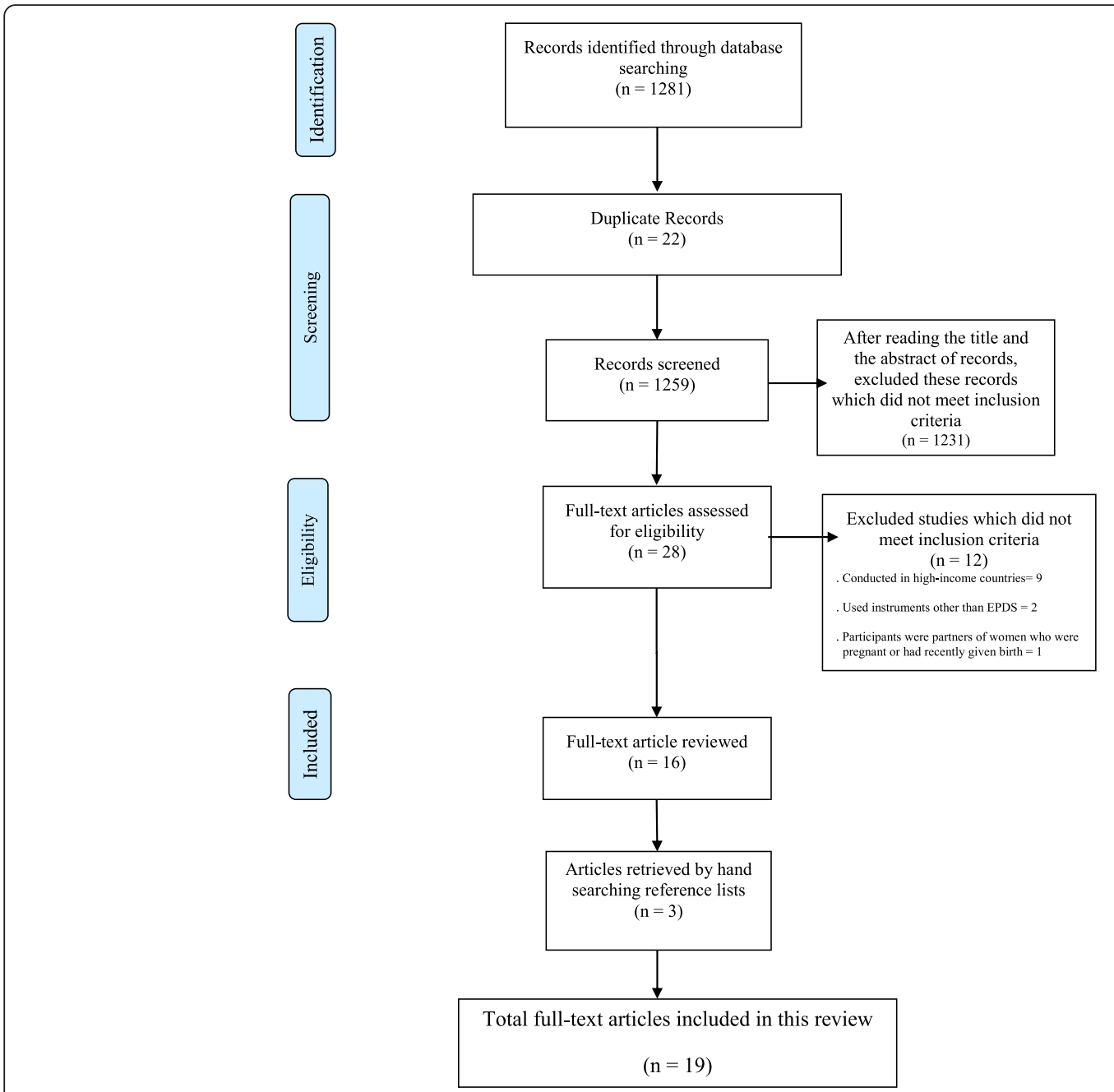

Fig. 2 Selection of studies for review on cultural adaptation and validation of the EPDS in low- and lower-middle-income countries (defined as per World Bank criteria)

translators were also included $[29,30]$. Only one study used a committee approach, which included lay-people in addition to multi-disciplinary health professionals, and there were a number of meetings during the translation process [19].

Nine studies reported pre-testing the preliminary versions of the LLV-EPDS, however only four of these studies (involving the Amharic version from Ethiopia, along with the Bangla and Vietnamese versions) [19, 23, 33, 35] probed understanding and comprehension among women who had recently given birth. One type of amendment in these three LLV-EPDS translations was in the format of the instrument. For example, in the Bangla version to enable administration by an interviewer for respondents who were illiterate, women were addressed in the second person ('you'). To reduce repetition, the short reply statements were replaced by numerals [19]. In the Amharic version, all ten items were changed into a question. The mode of response was changed into two stages: first by asking a fixed choice Yes/No question, then in the next step probing the frequency and severity of the reported symptoms. Additionally, the short reply statements were rephrased for clarity. Still more, to remind study participants about the timeframe the phrase "last week" was added at the end of the each item [33].

The other type of amendment aimed to establish semantic equivalence of the LLV-EPDS in the local context. Altogether four studies [19, 23, 33, 35] altered 8/10 EPDS items in this manner. Among three LLVEPDS (Amharic, Bangla and Vietnamese) versions, we found an overlap among items modified in the different versions, but there were also items that were modified in one study but not in others. In a study conducted in Ethiopia, Hanlon et al. [33] modified six items in the Amharic version (1-5 \& 9), and to ease understanding by rural women including illustrative examples for items 1-3. Despite these modifications, in a re-validation of this version with urban-dwelling women who had recently 
Table 2 Methodological quality of studies on translation and validation of the Edinburgh Postnatal Depression Scale in low- and lower-middle-income countries

\begin{tabular}{|c|c|c|c|c|c|c|c|c|c|c|}
\hline Study & $\begin{array}{l}\text { Clear } \\
\text { study aim }\end{array}$ & $\begin{array}{l}\text { Sample } \\
\text { adequacy } \\
\text { (justification) }\end{array}$ & $\begin{array}{l}\text { Representative } \\
\text { sample (with } \\
\text { justification) }\end{array}$ & $\begin{array}{l}\text { Explicit criteria } \\
\text { for inclusion } \\
\& \text { exclusion }\end{array}$ & $\begin{array}{l}\text { Response } \\
\text { rate }\end{array}$ & $\begin{array}{l}\text { Description } \\
\text { of data }\end{array}$ & $\begin{array}{l}\text { Appropriate } \\
\text { statistical } \\
\text { analyses } \\
\end{array}$ & $\begin{array}{l}\text { Ethics } \\
\text { approval }\end{array}$ & $\begin{array}{l}\text { Obtained informed } \\
\text { consent from } \\
\text { participants }\end{array}$ & $\begin{array}{l}\text { Total } \\
\text { score }\end{array}$ \\
\hline $\begin{array}{l}\text { 1. Nepal et al., } \\
\text { (2002) [24] }\end{array}$ & 1 & 0 & 0 & 1 & 1 & 1 & 0 & 0 & 0 & 4 \\
\hline $\begin{array}{l}\text { 2. Regmi et al., } \\
\text { (2002) [40] }\end{array}$ & 1 & 0 & 0 & 1 & 1 & 0 & 1 & 1 & 0 & 5 \\
\hline $\begin{array}{l}\text { 3. Patel et al., } \\
\text { (2002) [28] }\end{array}$ & 1 & 0 & 0 & 1 & 1 & 1 & 1 & 0 & 1 & 6 \\
\hline $\begin{array}{l}\text { 4. Uwakwe } \\
\text { (2003) [30] }\end{array}$ & 1 & 0 & 0 & 1 & 1 & 1 & 1 & 0 & 0 & 5 \\
\hline $\begin{array}{l}\text { 5. Fisher et al., } \\
\text { (2004) [23] }\end{array}$ & 1 & 1 & 1 & 1 & 1 & 1 & 1 & 1 & 0 & 8 \\
\hline $\begin{array}{l}\text { 6. Rahman } \\
\text { et al., } \\
\text { (2005) [39] }\end{array}$ & 1 & 1 & 1 & 1 & 1 & 1 & 1 & 1 & 0 & 8 \\
\hline $\begin{array}{l}\text { 7. Adewuya } \\
\text { et al., } \\
\text { (2006) [31] }\end{array}$ & 1 & 0 & 0 & 1 & 0 & 1 & 1 & 1 & 0 & 5 \\
\hline $\begin{array}{l}\text { 8. Pollock } \\
\text { et al., } \\
\text { (2006) [32] }\end{array}$ & 1 & 0 & 0 & 1 & 1 & 0 & 1 & 1 & 1 & 6 \\
\hline $\begin{array}{l}\text { 9. Gausia } \\
\text { et al., (2007) } \\
\text { [19] }\end{array}$ & 1 & 0 & 1 & 1 & 1 & 1 & 1 & 1 & 0 & 7 \\
\hline $\begin{array}{l}\text { 10. Gausia } \\
\text { et al., } \\
\text { (2007) [25] }\end{array}$ & 1 & 0 & 1 & 1 & 0 & 1 & 1 & 1 & 0 & 6 \\
\hline $\begin{array}{l}\text { 11. Rowel } \\
\text { et al., (2008) } \\
\text { [37] }\end{array}$ & 1 & 1 & 1 & 1 & 0 & 0 & 1 & 0 & 0 & 5 \\
\hline $\begin{array}{l}\text { 12. Hanlon } \\
\text { et al., } \\
\text { (2008) [33] }\end{array}$ & 1 & 0 & 1 & 0 & 1 & 1 & 1 & 1 & 0 & 6 \\
\hline $\begin{array}{l}\text { 13. Weobong } \\
\text { et al., } \\
\text { (2009) [38] }\end{array}$ & 1 & 0 & 1 & 0 & 0 & 1 & 1 & 0 & 0 & 4 \\
\hline $\begin{array}{l}\text { 14. Tesfaye } \\
\text { et al., (2010) } \\
\text { [35] }\end{array}$ & 1 & 0 & 1 & 0 & 1 & 1 & 1 & 1 & 0 & 6 \\
\hline $\begin{array}{l}\text { 15. Chibanda } \\
\text { et al., } \\
\text { (2010) [36] }\end{array}$ & 1 & 0 & 0 & 1 & 1 & 1 & 1 & 1 & 1 & 7 \\
\hline $\begin{array}{l}\text { 16. Fernandes } \\
\text { et al. } \\
\text { (2010) [29] }\end{array}$ & 1 & 0 & 0 & 1 & 0 & 0 & 1 & 1 & 0 & 4 \\
\hline $\begin{array}{l}\text { 17. Tran et al., } \\
\text { (2011) [27] }\end{array}$ & 1 & 1 & 1 & 1 & 0 & 1 & 1 & 1 & 0 & 7 \\
\hline $\begin{array}{l}\text { 18. Husain } \\
\text { et al., } \\
\text { (2013) [26] }\end{array}$ & 1 & 1 & 0 & 1 & 1 & 0 & 1 & 1 & 0 & 6 \\
\hline $\begin{array}{l}\text { 19. Stewart } \\
\text { et al., } \\
\text { (2013) [34] }\end{array}$ & 1 & 0 & 0 & 1 & 0 & 1 & 1 & 1 & 0 & 5 \\
\hline
\end{tabular}


Table 3 Culturally Sensitive Translation of the Edinburgh Postnatal Depression Scale in 12 low -and lower-middle-income countries

\begin{tabular}{|c|c|c|c|c|c|c|c|}
\hline $\begin{array}{l}\text { Country } \\
\text { and Author }\end{array}$ & Forward translation & $\begin{array}{l}\text { Back } \\
\text { translation }\end{array}$ & $\begin{array}{l}\text { Resolution of differences } \\
\text { in translations by } \\
\text { Committee approach }\end{array}$ & Pretesting & Amendments & $\begin{array}{l}\text { Test of } \\
\text { Equivalence }\end{array}$ & $\begin{array}{l}\text { Translated } \\
\text { version }\end{array}$ \\
\hline \multicolumn{8}{|l|}{ 1. Nepal } \\
\hline $\begin{array}{l}\text { Nepal et al., } \\
\text { (1999) [24] }\end{array}$ & Yes (psychiatrist) & $\begin{array}{l}\text { Yes (Another } \\
\text { set) }\end{array}$ & NM & Yes & Not needed & NM & Nepalese \\
\hline \multicolumn{8}{|l|}{ 2. India } \\
\hline $\begin{array}{l}\text { Patel et al., } \\
\text { (2002) [28] }\end{array}$ & Yes & Yes & NM & NM & NM & NM & Konkani \\
\hline $\begin{array}{l}\text { Fernandes et al. } \\
\text { (2010) [29] }\end{array}$ & $\begin{array}{l}\text { Yes ( } 1 \text { health } \\
\text { professional) }\end{array}$ & $\begin{array}{l}\text { Yes (Another } \\
\text { set: health } \\
\text { professional) }\end{array}$ & $\begin{array}{l}\text { Yes (investigator, } \\
\text { translators \& another } \\
\text { member of the } \\
\text { study team) }\end{array}$ & Yes & NM & NM & Kannada \\
\hline \multicolumn{8}{|l|}{ 3. Nigeria } \\
\hline $\begin{array}{l}\text { Uwakwe } \\
\text { (2003) [30] }\end{array}$ & Yes (3 nurses) & $\begin{array}{l}\text { Yes (Another } \\
\text { set: } 2 \text { medical } \\
\text { students \& } \\
\text { layperson) }\end{array}$ & $\begin{array}{l}\text { Yes (investigator } \\
\text { \&translators) }\end{array}$ & Yes & Not needed & NM & $\begin{array}{l}\text { lgbo } \\
\text { (Eastern) }\end{array}$ \\
\hline $\begin{array}{l}\text { Adewuya et al., } \\
\text { (2006) [31] }\end{array}$ & $\begin{array}{l}\text { Yes (Psychiatrist } \\
\text { \& linguist) }\end{array}$ & $\begin{array}{l}\text { Yes (Another } \\
\text { set: psychiatrist } \\
\text { \& linguist) }\end{array}$ & NM & NM & NM & NM & $\begin{array}{l}\text { Yoruba } \\
\text { (Western) }\end{array}$ \\
\hline \multicolumn{8}{|l|}{ 4. Pakistan } \\
\hline $\begin{array}{l}\text { Rahman et al., } \\
\text { (2005) [39] }\end{array}$ & Yes & Yes & IYes & NM & NM & NM & Urdu \\
\hline \multicolumn{8}{|l|}{ 5. Mongolia } \\
\hline $\begin{array}{l}\text { Pollock et al., } \\
\text { (2006) [32] }\end{array}$ & $\begin{array}{l}\text { Yes ( } 2 \text { medical } \\
\text { translators) }\end{array}$ & $\begin{array}{l}\text { Yes (Another } \\
\text { set: } 2 \text { medical } \\
\text { translators) }\end{array}$ & $\begin{array}{l}\text { Yes (investigator, } \\
\text { psychiatrist \& doctor) }\end{array}$ & NM & Yes & NM & Mongolian \\
\hline \multicolumn{8}{|l|}{ 6. Bangladesh } \\
\hline $\begin{array}{l}\text { Gausia et al., } \\
\text { (2007) [19] }\end{array}$ & $\begin{array}{l}\text { Yes (1 Principal } \\
\text { investigator) }\end{array}$ & $\begin{array}{l}\text { Yes (Another } \\
\text { set: translator } \\
\& 2 \text { native } \\
\text { English } \\
\text { speakers) }\end{array}$ & $\begin{array}{l}\text { Yes (investigator,1 } \\
\text { psychologist, 1 } \\
\text { psychiatrist, 1 } \\
\text { paediatrician, } 3 \\
\text { physicians, 2 } \\
\text { lay persons) }\end{array}$ & Yes (Probing) & Yes & Yes & Bengali \\
\hline \multicolumn{8}{|l|}{ 7. Sri Lanka } \\
\hline $\begin{array}{l}\text { Rowel et al., } \\
\text { (2008) [37] }\end{array}$ & Yes & Yes & NM & Yes & NM & NM & Sinhalese \\
\hline \multicolumn{8}{|l|}{ 8. Ethiopia } \\
\hline $\begin{array}{l}\text { Hanlon et al., } \\
\text { (2008) [33] }\end{array}$ & $\begin{array}{l}\text { Yes (Physicians) Yes } \\
\text { (Another set: physicians) }\end{array}$ & & $\begin{array}{l}\text { Yes (investigator,2 } \\
\text { senior psychiatrists) }\end{array}$ & Yes (Probing) & Yes & NM & Amharic \\
\hline $\begin{array}{l}\text { Tesfaye et al., } \\
\text { (2010) [35] }\end{array}$ & $\begin{array}{l}\text { Re-validation of the earlier } \\
\text { EPDS -Amharic version } \\
\text { (Hanlon et al., [33]) }\end{array}$ & & NM & Yes (Probing) & Yes & NM & \\
\hline \multicolumn{8}{|l|}{ 9. Ghana } \\
\hline $\begin{array}{l}\text { Weobong et al., } \\
\text { (2009) [38] }\end{array}$ & $\begin{array}{l}\text { Yes (Native \& UK } \\
\text { professionals) }\end{array}$ & $\begin{array}{l}\text { Yes (Native \& } \\
\text { UK } \\
\text { professionals) }\end{array}$ & Yes (study team) & (Qualitative study) & Not needed & NM & Twi \\
\hline \multicolumn{8}{|l|}{ 10. Zimbabwe } \\
\hline Chibanda et al., & Yes (research assistant) & Yes & Yes (study team) & NM & NM & NM & Shona \\
\hline
\end{tabular}


Table 3 Culturally Sensitive Translation of the Edinburgh Postnatal Depression Scale in 12 low -and lower-middle-income countries (Continued)

\begin{tabular}{|c|c|c|c|c|c|c|c|}
\hline \multicolumn{8}{|l|}{ 11. Vietnam } \\
\hline $\begin{array}{l}\text { fFisher et al., } \\
\text { (2004) [23] }\end{array}$ & Yes & Yes & Yes (study team) & Yes (Probing) & Yes & NM & $V_{i e t n a m e s e}{ }^{a}$ \\
\hline \multicolumn{8}{|l|}{ 12. Malawi } \\
\hline $\begin{array}{l}\text { Stewart et al., } \\
\text { (2013) [34] }\end{array}$ & $\begin{array}{l}\text { Yes ( } 1 \text { health professional, } \\
\text { psychiatrist (UK), } 2 \text { social } \\
\text { science graduates) }\end{array}$ & $\begin{array}{l}\text { Yes (Another } \\
\text { set: } 1 \text { non- } \\
\text { mental health } \\
\text { professional) }\end{array}$ & $\begin{array}{l}\text { Yes (antenatal } \\
\text { clinic nurses) }\end{array}$ & Yes & Yes & NM & $\begin{array}{l}\text { Chichewa } \\
\text { ('Visual } \\
\text { Prompt card }\end{array}$ \\
\hline
\end{tabular}

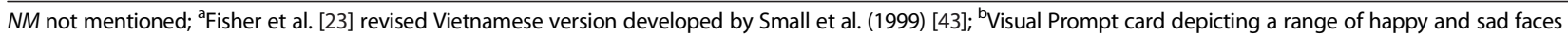
was used along with the Chichewa-version-EPDS

given birth, Tesfaye et al. [35] found that items 1 and 2 were still not understood and that provision of examples did not help respondents to understand these question. Connotative translations using local expression were reported for item 6 for the Vietnamese [23] and the Bangla [19] versions. Similarly, item 10 (relating to suicidal ideation) was rephrased in the Vietnamese version as the content was ambiguous in translation [23]. In Ethiopia mixed responses were found; women in rural areas were open to the question about ideas of self harm [33], while women in urban areas without this symptom were embarrassed to be asked it, but women who reported had suicidal ideas reported relief at the interviewer asking this question [35].

The tests of conceptual and operational equivalence of these LLV-EPDS with the original English version was performed only for the Bangla version EPDS. The test showed a higher conceptual equivalence, with the original English EPDS (correlation coefficient 0.981; $p<0.01)$. The operational equivalence test, which is to investigate whether the local version can achieve similar outcomes when administered by self-report as by an interviewer was slightly lower (correlation coefficient $0.752 ; p=0.01$ ) [19].

\section{Empirical Validation}

There were 17 studies on psychometric validations of 14 LLV-EPDS from 12 countries; due to inclusion of two studies from each of five countries: Ethiopia [33, 35], India [28, 29], Nepal [24, 40], Nigeria [30, 31] and Pakistan [26, 39] (Table 4).

Generation of data on performance of these 14 LLVEPDS was mainly carried out by recruiting study participants from ante- or postnatal or immunisation clinics (13/17 studies) [24, 25, 28-32, 34, 36-38, 40]. More than half of the studies (10/17) recruited women who had recently given birth ( 0 to 36 months ago) [24-26, 28, 30, $33,35,36,39,40]$; while $4 / 17$ studies included women who were currently pregnant $[29,31,34,38]$; there were both pregnant women and those who had recently given birth in two studies [27, 37] and in one study all participants were women of reproductive age (18-40 years) [32]. Of the 11 publications [24, 25, 27-29, 31, 32, 34-36, 39] that mentioned participants' literacy status, almost all had a predominance of literate participants (67 to $89 \%$ ), except in a rural community-based investigation from Pakistan, in which only a quarter of women could read [39]. In 10/17 studies, interviewers administered the LLV-EPDS [25, 26, 28, 29, 31, 33, 35, 36, 38, 39].

Diagnostic interviews were carried out to establish clinical cut-off points. Of the 17 studies, in 11studies all participants who were screened using LLV-EPDS were also recruited for diagnostic interviews. In three studies, all those scoring higher than certain scores $(\geq 6, \geq 9$ and $\geq 13)$ and a randomly selected sample of woman who scored below the cut-offs were included. In the remaining two studies, about half the participants were included (Table 4).

Psychiatrists or psychologists carried out the diagnostic interviews in $12 / 17$ studies, but in eight of these publications, it is not clear if they were blinded to the initial screening results [24, 28-30, 32, 33, 37, 40]. In addition, in eight studies [24, 28-31, 33, 37, 40] it is not clear whether the diagnostic interviews were carried out on the same day as the screening. Furthermore, although interviews were conducted in the local language, the standard diagnostic protocol (SDP) were translated into respective local languages in only three studies [29, 32, 39], while only two of the SDP had been culturally adapted [32, 39]. In $6 / 17$ studies, there was more than one diagnostic interviewer; but only one of these investigated interrater reliability and reported excellent reliability between psychiatrists $($ kappa $=0.82)$ [33].

\section{Psychometric Properties}

The 17 studies of the 14 LLV-EPDS revealed wide variation in their psychometric properties. The range of cut-off scores selected for detecting any common mental disorder was $3 / 4$ to $11 / 12$; and where specific conditions were listed, the range of cut-off scores was 3/4 [27] to 13/14 [26] for depression (mild and moderate) and 4/5 


\begin{tabular}{|c|c|c|c|c|c|c|c|c|c|c|c|c|}
\hline $\begin{array}{l}\text { Country and } \\
\text { Author }\end{array}$ & Participant characteristics & $\begin{array}{l}\text { Mode of } \\
\text { administration }\end{array}$ & Sub-sample /Sample & Same day & Blinded & Administered by & $\begin{array}{l}\text { Diagnostic Instruments }{ }^{c} \\
\& \text { diagnostic criteria }\end{array}$ & Cut-off & $\begin{array}{l}\mathrm{Se}^{\mathrm{e}} \\
(\%)\end{array}$ & $\begin{array}{l}\mathrm{Sp}^{f} \\
(\%)\end{array}$ & $\begin{array}{l}\text { PPV }^{9} \\
(\%)\end{array}$ & $\begin{array}{l}\text { NPVh } \\
(\%)\end{array}$ \\
\hline \multicolumn{13}{|l|}{ Nepal } \\
\hline $\begin{array}{l}\text { Nepal et al., } \\
\text { (1999) [24] }\end{array}$ & $\begin{array}{l}132 / 149 \text { postnatal women } \\
\text { convenientlyrecruited from } \\
\text { the maternity wards of two } \\
\text { hospitals ( } \geq 2 \text { days post- } \\
\text { delivery) located in the } \\
\text { Kathmandu. Then followed } \\
\text { up after } 4 \text { weeks ( } 84.5 \% \\
\text { were literate). }\end{array}$ & Interview & All 132 participants & NM & NM & 2 Psychiatrists & $\begin{array}{l}\text { DSM-IV of major } \\
\text { depression }\end{array}$ & $12 / 13$ & 68.4 & 93.8 & 65 & 94.6 \\
\hline $\begin{array}{l}{ }^{a} \text { Regmi et al., } \\
\text { (2002) [40] }\end{array}$ & $\begin{array}{l}100 \text { postnatal women ( } 2-3 \\
\text { months) conveniently } \\
\text { recruited from the post- } \\
\text { natal clinic of the university } \\
\text { teaching hospital in the } \\
\text { Kathmandu. This case- } \\
\text { controlled study recruited } 40 \\
\text { non-child bearing women } \\
\text { as controls (mainly nurses \& } \\
\text { their friends). }\end{array}$ & Self-reporting & $\begin{array}{l}30 \text { postpartum } \\
\text { women (all } 12 \\
\text { scored } \geq 13 \text { and } \\
\text { rest scored } \leq 12 \\
\text { were randomly } \\
\text { selected) }\end{array}$ & NM & NM & NM & $\begin{array}{l}\text { SCID DSM-IV of } \\
\text { major depression }\end{array}$ & & 100 & 92.6 & 41.6 & 100 \\
\hline \multicolumn{13}{|l|}{ India } \\
\hline $\begin{array}{l}\text { Patel et al., } \\
\text { (2002) [28] }\end{array}$ & $\begin{array}{l}270 / 297 \text { pregnant }(\geq 30 \\
\text { week) women conveniently } \\
\text { recruited from antenatal } \\
\text { clinics, and then followed } \\
6-8 \text { weeks after delivery } \\
\text { (252), in Goa. Konkani, } \\
\text { Marathi, Hindi and English } \\
\text { speakers were included for } \\
\text { this study. In this state, the } \\
\text { female literacy rate is } 67 \% \\
\text { and } 87 \% \text { of births } \\
\text { are supervised. }\end{array}$ & Interviewers & Not clear & NM & NM & NM & $\begin{array}{l}\text { CIS-R of common } \\
\text { mental disorders }\end{array}$ & $11 / 12$ & 92 & 85 & NM & NM \\
\hline $\begin{array}{l}\text { Fernandes et al. } \\
\text { (2010) [29] }\end{array}$ & $\begin{array}{l}\text { 194/196 pregnant ( } 32-38 \\
\text { weeks) women conveniently } \\
\text { recruited from the antenatal } \\
\text { clinic of the missionary h } \\
\text { ospital located in the rural } \\
\text { area of Karnataka state. } \\
95.5 \% \text { of these women had } \\
\text { completed primary education. }\end{array}$ & Interviewer & All 194 & Yes & NM & 1 psychologist & $\begin{array}{l}\text { MINI DSM-IV of } \\
\text { depression } \\
\text { (translated) }\end{array}$ & $12 / 13$ & 100 & 84.9 & 52 & 99 \\
\hline
\end{tabular}


Table 4 Empirical Validation of the local language versions of the Edinburgh Postnatal Depression Scale in 12 low- and- lower-middle-income countries (Continued)

\section{Nigeria}

Uwakwe

(2003) [30]

225/292 postnatal women conveniently recruited from

the maternity ward $(\geq 7$

days post-delivery), of

teaching hospital \&

postnatal clinic.

Adewuya et al., 182 pregnant women ( $\geq 32$ (2006) [31]

weeks) conveniently

recruited from the antenata

clinics of 5 health centres,

located in a semi-urban

town of western Nigeria

(15.4\% were illiterate).

Pakistan

Rahman et al., $\quad 541 / 570$ postnatal women

(10 to12 weeks) recruited

from a rural community of

from a rural community of

About $75 \%$ were illiterate.

Husain et al., $\quad 601 / 664$ postnatal women

(2013) [26] (0-36 months) recruited

from an urban slum in the

capital Karachi.

Mongolia

Pollock et al., (2006) [32]

94/100 women (in

reproductive age) conveniently

recruited from two specialised

psychiatric units (55) \& rest

from the 3 community based

immu1nization clinics in the

capital Ulaanbaatar (adult

literacy rate near $100 \%$ )

Bangladesh

Gausia et al., $\quad$ 100/126 postnatal women

(2007) [25]

(6-8 weeks) conveniently

recruited from a child

immunization clinic in

Dhaka, $11 \%$ were illiterate.
Self-reported, $\quad 94.0 \%$ of the NM

using English or participants

ocal-version EPDS.

Psychiatrist \&

Diagnostic

interview using

ICD -10 for

mental disorders

Interviewer

administered

for illiterate

86 (all 75 scored $\geq 6$

\& rest $10 \%$ randomly

selected out of those

women. scoring $<6$ )

Interviewers

All 541

Yes

2 Mental health
professionals

SCAN for ICD-10

for depressive

disorders

(translated \&

adapted)

All 601

Yes

Yes

NM

CIS-R, ICD $-10^{b}$

for depression

All 94

NM

1 bstetrician-an/

CIS-R, ICD-10 for

depressive

disorders

\& adapted

1 interviewer

All 100

Yes

1 psychiatrist

SCID DSM-IV

of depression $\begin{array}{llll}8 / 9 & 75 & 97 & 75\end{array}$

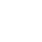


Table 4 Empirical Validation of the local language versions of the Edinburgh Postnatal Depression Scale in 12 low- and- lower-middle-income countries (Continued)

\begin{tabular}{|c|c|c|c|c|c|c|c|c|c|c|c|c|}
\hline \multicolumn{9}{|l|}{ Sri Lanka } & \multicolumn{4}{|c|}{ Pregnant } \\
\hline \multirow{3}{*}{$\begin{array}{l}\text { Rowel et al., } \\
\text { (2008) [37] }\end{array}$} & \multirow{3}{*}{$\begin{array}{l}465 \text { perinatal women } \\
\text { conveniently recruited for } \\
\text { this study: of them } 265 \\
\text { were pregnant ( } \geq 34 \text { weeks) } \\
\text { and attending antenatal } \\
\text { clinics. The other } 204 \text { were } \\
\text { postpartum women ( } \geq 6 \\
\text { weeks) attending a family } \\
\text { planning or child wellbeing } \\
\text { clinic (all could read \& write). }\end{array}$} & \multirow[t]{3}{*}{ Not clear } & \multirow[t]{3}{*}{ All 465} & \multirow[t]{3}{*}{ NM } & \multirow[t]{3}{*}{ NM } & \multirow[t]{3}{*}{1 psychiatrist } & \multirow{3}{*}{$\begin{array}{l}\text { Diagnostic } \\
\text { interview using } \\
\text { ICD-10 for } \\
\text { mental disorders }\end{array}$} & \multirow[t]{3}{*}{$8 / 9$} & 90.7 & 86.8 & NM & NM \\
\hline & & & & & & & & & \multicolumn{4}{|c|}{ Postnatal } \\
\hline & & & & & & & & & 89.9 & 78.9 & NM & NM \\
\hline \multicolumn{13}{|l|}{ Ethiopia } \\
\hline $\begin{array}{l}\text { Hanlon et al., } \\
\text { (2008) [33] }\end{array}$ & $\begin{array}{l}101 \text { postnatal women } \\
\text { (median } 5 \text { Months) } \\
\text { recruited from the Butajra } \\
\text { sub-district (rural community) }\end{array}$ & 20 Interviewers & 52 participants & NM & NM & Psychiatrists & $\begin{array}{l}\text { CPRS DSM-IV of } \\
\text { common mental } \\
\text { disorders }\end{array}$ & $5 / 6$ & 76.5 & 36.1 & NM & NM \\
\hline $\begin{array}{l}\text { Tesfaye et al., } \\
\text { (2010) [35] }\end{array}$ & $\begin{array}{l}100 / 102 \text { postnatal women } \\
(6 \text { to } 14 \text { weeks) conveniently } \\
\text { recruited from child } \\
\text { immunization and/or } \\
\text { postnatal clinics in } 2 \text { primary } \\
\text { health care centres, located } \\
\text { in the peri-urban area of the } \\
\text { capital Addis Ababa. } 21 \% \\
\text { were illiterate. }\end{array}$ & Interviewers & All 100 & Yes & Yes & 2 psychiatrists & & $6 / 7$ & 78.9 & 75.3 & 42.9 & 93.8 \\
\hline \multicolumn{13}{|l|}{ Ghana } \\
\hline $\begin{array}{l}\text { Weobong et al., } \\
\text { (2009) [38] }\end{array}$ & $\begin{array}{l}160 \text { pregnant women }(5-11 \\
\text { week) identified from the } \\
\text { database of } 1 / 6 \text { districts } \\
\text { where vitamin } A \text { trial was } \\
\text { implemented. }\end{array}$ & Interviewers & About half & Yes & Yes & 1 psychologist & $\begin{array}{l}\text { SCAN for common } \\
\text { mental disorders }\end{array}$ & $10 / 11$ & 78 & 73 & 22 & 97 \\
\hline \multicolumn{13}{|l|}{ Zimbabwe } \\
\hline $\begin{array}{l}\text { Chibanda et al., } \\
\text { (2010) [36] }\end{array}$ & $\begin{array}{l}210 / 223 \text { postnatal women } \\
(6-7 \text { weeks) conveniently } \\
\text { recruited from the } 2 \text { primary } \\
\text { health care centres, located } \\
\text { in a peri-urban area of the } \\
\text { capital Harare ( } 74 \% \text { completed } \\
\text { secondary education). }\end{array}$ & 6 interviewers & All 210 & Yes & Yes & 2 psychiatrists & $\begin{array}{l}\text { DSM-IV of major } \\
\text { depression }\end{array}$ & $10 / 11^{b}$ & 88 & 87 & 74 & 94 \\
\hline
\end{tabular}


Table 4 Empirical Validation of the local language versions of the Edinburgh Postnatal Depression Scale in 12 low- and- lower-middle-income countries (Continued)

Vietnam

Tran et a

(2011) [27]

364/392 perinatal women

(199 were $\geq 28$ weeks

Interviewers

All 364

Yes

Yes

SCID DSM-IV of

depression,

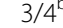

697

$\begin{array}{ll}72.9 & 69.7\end{array}$

pregnant \& rest were 4-6

randomly selected commune

health centres in the capital

Hanoi. Rural women were

recruitedfrom the $\mathrm{Ha}$

Nam province.

Malawi

(2013)

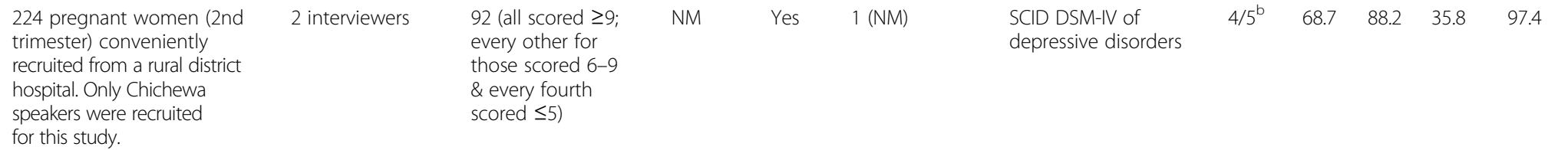

for this study.

aWile the validation of a Nepalese version EPDS was published by Nepal et al., [24], it is not clear that particular Nepalese version was used by Regmi et al. [40];

NM not mentioned: ${ }^{\mathrm{b}}$ of the multiple cut-offs, presented one that had Sensitivity and Specificity nearest to $80 \%$;

'Diagnostic Instrument: SCID Structured Clinical Interview for DSM, CIS-R Revised Clinical Interview Schedule, MINIPLUS Mini International Neuropsychiatric Interview, SCAN Schedule for Assessment in Neuropsychiatry,

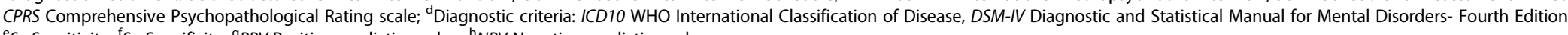

${ }^{\mathrm{e}} \mathrm{S}$ Sensitivity, ${ }^{\mathrm{A} S p}$ Specificity, ${ }^{9}$ PPV Positive predictive value, ${ }^{\text {, } N P V ~ N e g a t i v e ~ p r e d i c t i v e ~ v a l u e ~}$ 
to $12 / 13$ for severe depression [40]. The scores for sensitivity (identifying true cases) ranged from $69.7 \%$ [27] up to $100 \%[29,40]$. The scores for specificity (identifying those without PCMDs) ranged from $36.1 \%$ [33] up to $97 \%$ [30]. The positive predictive value (PPV, which is the proportion of respondents who scoring positive in the screening, who were confirmed to have a common mental disorder by clinical interview) ranged from $22 \%$ [38] up to $82 \%$ [26]; the negative predictive value (NPV, which is the proportion of the respondents scoring negative in the screening who were confirmed as having no mental disorders) ranged from $70 \%$ [26] to $100 \%$ [40]. The range of cut-off points for detecting depression among pregnant women was slightly lower $4 / 5$ [34] to $12 / 13$ [29] than among women who had recently given birth $5 / 6$ [33] to $13 / 14$ [26].

\section{Discussion}

To our knowledge, this systematic review is the first assessment of EPDS versions translated and adapted for use in low- and lower-middle-income countries (LALMICs). Additionally, this is the first study to use a new set of process-based criteria (Fig. 1) to assess the reliability and validity of LLV-EPDS comprehensively. We acknowledge the possible limitation of this review, that some studies on LLV-EPDS may have been missed, if they had been published in languages other than English in non-indexed journals. However, we think this is unlikely, because we followed rigorous strategies to identify pertinent studies published in non-indexed journals.

We found that of the 82 countries classified by the World Bank (in 2015) as LALMICs, the Edinburgh Postnatal Depression Scale (EPDS) had been formally validated in $12(14.6 \%)$ countries in 14 native languages. We found psychometric properties of these 14 LLVEPDS from low-and middle-income countries were lower than that for the original English EPDS [6]. Our finding is consistent with findings from earlier three systematic reviews that included studies from high-, middle- and low-income countries [3, 4] and African countries [12].

A central finding of our systematic review is that utility of these 14 local versions EPDS for screening PCMDs is questionable, as none met the recommended validation standard of $\geq 80 \%$ in the three key parameters: sensitivity, specificity and positive predictive value [41]. The process-based appraisal indicated that lower psychometric property of these LLV-EPDS might be related to compromises made during translation, cultural adaptation and empirical validation (Tables 3 and 4). The psychometric properties of the LLV-EPDS were found to be better when the process-based criteria were followed. In Ethiopia, Tesfaye et al. [35] achieved an almost two fold improvement in specificity ( $75.3 \%$ vs. $36.1 \%)$, by inclusion of more suitable local expressions than in the earlier Amharic version EPDS [33]. The Bangla EPDS, was the only one which met all steps and criteria for culturally sensitive translation, cultural adaptation and empirical validation and was the one study that demonstrated high sensitivity (88.9 \%) and specificity $(86.8 \%)$ [19] (Table 4).

Sub-optimal sensitivity and specificity in these LLVEPDS might also have been attributable to the recruitment of women who did not represent the wider perinatal population during empirical validation (Fig. 1, criteria: 15-21). For instance, more than half of the studies (10/17) recruited participants from health facilities, most usually a single, urban health institution, using convenience sampling. This is especially problematic in settings where many women give birth in primary care facilities or at home. Selection bias is apparent as there was a predominance of well educated women in these study samples. While they might understand direct, literally translated LLV-EPDS, it is much less likely that women of low literacy or education will understand them. The link between non-random selection of participants from health institutions and a poorly translated LLV-EPDS is shown by the study from Nigeria. In that country, while about half of the female population (50.6\%) are illiterate [42], more than two thirds of the study participants who were recruited from antenatal clinics were highly educated (bankers, teachers/lecturers, big business owners and civil servants). In this study, the LLV-EPDS had high sensitivity $(86.7 \%)$ and specificity (91.5\%), even though it didn't meet most of our recommended steps for culturally sensitive translation processes [31]. This suggests that these highly educated participants having greater emotional literacy $[3,23]$ and familiarity with test-taking [7], but does not provide evidence that this LLV-EPDS will be useful for the majority who have not had opportunities for education and social participation.

Further areas of inconsistency and suboptimal practice appeared to have occurred during the process of formal validation against a diagnostic interview (Fig. 1, criteria: 22-28). In 11/17 studies the interviews were carried out by psychiatrists or psychologists. However, in 8 of these studies, diagnosis might have been influenced by changes in the interviewees' psychological state as the screening by LLV-EPDS and diagnostic interviews were not conducted on the same day $[24,28,30,31,33,34,37,40]$. Further, the diagnosis might have been biased by the psychiatrists or psychologists being aware of the screening results, as only 8 studies reported that they were blinded to the initial screening results. Moreover, even though interviews were carried out in local languages, the accuracy of both screening and diagnosis may be influenced by participants' limited understanding of English colloquialisms. Only four 
studies made amendments to the LLV-EPDS so that it was suitable for administration to participants with low literacy, and tried to attain semantic equivalence to the local context by using local expressions [19, 23, 33, 35]. For instance, the statement in the original EPDS "Life is getting on top of me" is intended to detect an experience in which a woman feels that the demands imposed on her, exceed her capacity to manage them. However, it was interpreted by some women in Vietnam as meaning literally that things were being placed on top of them as might occur during a flood or other natural disaster [23]. Although all diagnostic interviews were presumed to be conducted in local languages, only two reported that standardised diagnostic protocols had been culturally adapted. It is possible that in the other studies participants might have not understood and or mis-understood questions asked by clinicians [32, 39].

Conceptual equivalence was generally not established between the LLV-EPDS and the original English EPDS [10]. In half of these studies, the back-translations to English might have been influenced by knowledge of the original version, as both forward and back-translation were carried out by the same panels. Additionally, the back-translated English version was reviewed by nativeEnglish-speakers in only one study [19]. Conceptual and operational disparities between the original English version and the LLV-EPDS were not investigated and tests of equivalence were not performed.

There is considerable variation in cut-off points to detect clinically significant symptoms among the 14 LLV-EPDS from non-English speaking low- and lowermiddle-income countries. In general lower cut-off scores compared to the English version were found. This probably reflects differences in cultural norms about emotional expression and emotional literacy $[7,9,23]$. The EPDS, developed in Britain, reflects the psychiatric paradigm that experiences of low mood are episodic and represent change from a usual state. It is inaccurate to presume that this is a universal situation. In resource constrained settings, where many women experience chronic social and economic adversity it is probable that they might not experience change from a usual state, but rather could be chronically distressed, so answers to questions about change would be negative [23]. There is also potential confusion, and perhaps linguistic limitations which mean that subtle emotional distinctions, for example being anxious or being scared might not be available or in widespread use and therefore lead to responses that do not reflect reality.

It would appear overall that formal validation of the EPDS following proposed process-based criteria is more likely to derive precise cut-off points appropriate to the local setting and /or population. Having a LLV-EPDS with an imprecise cut-off point has potentially serious implications. On one hand, an inaccurately high cut-off point imported from a high-income Anglophone setting might lead to under-detection of women with PCMDs. This means women's needs might go unrecognised and unassisted and lead to under-estimation of PCMDs burden for a particular country or population. On the other hand, if a cut-off point is too low, women might be classified as having a clinically significant condition of mental disorders which may lead to unnecessary treatment and potentially, stigma and discrimination, in particular in societies where experiences of human suffering are poorly understood. Inaccurate classification of 'cases' may further strain the already over-burdened health systems of low- and lower-middle-income countries [11].

\section{Conclusions}

It is commendable that researchers and clinicians in several resource-constrained countries have made great efforts to improve early detection and timely management of PCMDs. However, this review indicated that currently available local language versions of the Edinburgh Postnatal Depression scales (LLV-EPDS) from low-and lower- middle income countries are of some value, but most of them had deficiencies in translation, cultural

\footnotetext{
- The psychometric properties of LLV-EPDS are dependent on the processes used for translation, adaptation and validation.

- Accurate detection of perinatal common mental disorders using the EPDS crossculturally is premised on its comprehensibility to the general population of women, who are pregnant or have recently given birth. Effective and culturally sensitive translation and validation processes require explicit consideration of local terminology for psychological distress, mental disorders, emotional literacy and differences among people with different levels of literacy.

- In a multilingual country the EPDS should be translated, adapted and empirically validated in the national and minority languages.

- As the specificity and sensitivity of the EPDS varies between high-income Anglophone and other settings, only cut-off scores derived from empirically validated studies using culturally equivalent LLV-EPDS and appropriately culturally validated standard diagnostic protocol should be used.
}

Fig. 3 Recommendations for optimising psychometric properties of the LLV-EPDS in low- and lower-middle-income countries 
adaptation and validation processes. Screening instruments with poor psychometric properties might have far-reaching implications for clinical practice, public policy and research. We recommend a systematic approach to the translation, cultural adaptation and empirical validation of local language versions of the EPDS that adheres to the steps outlined in Fig. 3. This approach will facilitate the development of more precise and validated screening tools for detection and management of PCMDs among women in resource constrained settings.

\section{Additional files}

Additional file 1: Ten items of the Edinburgh Postnatal Depression Scale [5]. (DOCX $15 \mathrm{~kb})$

Additional file 2: PRISMA (2009) checklist for studies included for this systematic review. (DOCX $25 \mathrm{~kb}$ )

Additional file 3: Literature search strategy for systematic review on the reliability and validity of the EPDS in low and lower-middle income countries (LALMICs). (DOCX $14 \mathrm{~kb}$ )

Additional file 4: Definitions of the Process-based criteria. (DOCX 20 kb)

\section{Competing interests}

The authors declare that they have no competing interests.

\section{Authors' contributions}

SDS and JF designed the study. SDS conducted the systematic search, developed the novel process-based criteria and undertook the quality appraisal. JF and RG independently undertook the quality appraisal and analysis. SDS wrote the first draft of the manuscript. All authors contributed to revisions, read and approved the final manuscript.

\section{Acknowledgement}

The first author (SDS) acknowledges extension of her Adjunct Research Fellowship (after her injury in 2013) by Professor JF. She expresses her gratitude for the continuous encouragement and support provided by JF along with the Jean Hailes Research Unit towards regaining her academic ability, which made it possible to complete this manuscript.

\section{Funding}

SDS is an Adjunct Research Fellow in the Jean Hailes Research Unit, Monash University School of Public Health and Preventive Medicine. JF is supported by a Monash University Professional fellowship and the Jean Hailes Professional Fellowship, which receives funding from the $L$ and $\mathrm{H}$ Hecht trust which is administered by Perpetual Trustees Pty Ltd. TDT is supported by a Bridging Postdoctoral Research Fellowship from Monash University and an Early Career Fellowship from the Australian National Health and Medical Research Council.

\section{Author details}

${ }^{1}$ Ministry of Health and Population, Kathmandu, Nepal. ${ }^{2}$ Life Line Nepal, Kathmandu, Nepal. ${ }^{3}$ Jean Hailes Research Unit, Monash University School of Public Health and Preventive Medicine, Level 1, 549 St Kilda Road, Melbourne 3004, Victoria, Australia. ${ }^{4}$ Nepal Institute of Health Sciences, Affiliated Purbanchal University, Kathmandu, Nepal.

Received: 5 September 2015 Accepted: 23 March 2016 Published online: 04 April 2016

\section{References}

1. Fisher J, Cabral de Mello M, Patel V, Rahman A, Tran T, Holton S, Holmes W. Prevalence and determinants of common perinatal mental disorders in women in low-and lower-middle-income countries: A systematic review. Bull World Health Organ. 2012;90(2):139-49.
2. Rahman A, Fisher J, Bower P, Luchters S, Tran T, Yasamy MT, Saxena S, Waheed $\mathrm{W}$. Interventions for common perinatal mental disorders in women in low-and middle-income countries: A systematic review and meta-analysis. Bull World Health Organ. 2013:91(8):593-6011.

3. Gibson J, McKenzie MK, Shakespeare J, Price J, Gray R. A systematic review of studies validating the Edinburgh Postnatal Depression Scale in antepartum and postpartum women. Acta Psychiatr Scand. 2009;119(5):350-64.

4. Kozinszky Z, Dudas RB. Validation studies of the Edinburgh Postnatal Depression Scale for the antenatal period. J Affect Disord. 2015;176:95-105.

5. Cox J, Holden J. Perinatal Psychiatry: Use and misuse of the Edinburgh Postnatal Depression Scale. Britain: The Royal College of Psychiatrists; 1994.

6. Cox JL, Holden JM, Sagovsky R. Detection of postnatal depression. Development of the 10-item Edinburgh Postnatal Depression Scale. $\mathrm{Br} J$ Psychiatry. 1987;150(6):782-6.

7. Laungani P. Postnatal depression across cultures: conceptual and methodological considerations. Int J Health Promot Educ. 2000;38(3):86-94.

8. Shrestha SD. Quality of life of HIV-positive people undertaking antiretroviral therapy in Nepal: A mixed method study. Melbourne: La Trobe University; 2013.

9. Patel V, Abas M, Broadhead J, Todd C, Reeler A. Depression in developing countries: lessons from Zimbabwe. Br Med J. 2001;322(7284):482-4.

10. Wild D, Grove A, Martin M, Eremenco S, McElroy S, Verjee-Lorenz A, Erikson P. Principles of Good Practice for the Translation and Cultural Adaptation Process for Patient-Reported Outcomes (PRO) Measures: Report of the ISPOR Task Force for Translation and Cultural Adaptation. Value Health. 2005:8(2):94-104.

11. Matthey S, Henshaw C, Elliott S, Barnett B. Variability in use of cut-off scores and formats on the Edinburgh Postnatal Depression Scale-implications for clinical and research practice. Arch Womens Ment Health. 2006;9(6):309-15.

12. Tsai AC, Scott JA, Hung KJ, Zhu JQ, Matthews LT, Psaros C, Tomlinson M. Reliability and validity of instruments for assessing perinatal depression in African settings: Systematic review and meta-analysis. PLoS One. 2013;8(12):e82521.

13. Country and Lending Groups. [http://data.worldbank.org/about/ country-and-lending-groups]. Accessed April 2015.

14. Moher D, Liberati A, Tetzlaff J, Altman DG. Preferred reporting items for systmatic reviews and meta-analyses: The PRISMA statement. Br Med J. 2009;339:2535.

15. Mirza I, Jenkins R. Risk factors, prevalence, and treatment of anxiety and depressive disorders in Pakistan: systematic review. Br Med J. 2004; 328(7443):794.

16. Bowden A, Fox-Rushby JA. A systematic and critical review of the process of translation and adaptation of generic health-related quality of life measures in Africa, Asia, Eastern Europe, the Middle East. South Am Soc Sci Med. 2003;57(7):1289-306

17. Beusenberg M. A User's quide to the Self reporting questionnaire (SRQ). Geneva: Division of Mental Health, World Health Organization; 1994

18. Beck $C T$, Bernal $H$, Froman RD. Methods to document semantic equivalence of a translated scale. Res Nurs Health. 2003;26(1):64-73.

19. Gausia K, Hamadani JD, Islam MM, Ali M, Algin S, Yunus M, Fisher C, Oosthuizen J. Bangla translation, adaptation and piloting of Edinburgh Postnatal Depression Scale. Bangladesh Med Res Counc Bull. 2007;33(3):81-7.

20. Department of Health Government of Western Australia. Using the Edinburgh Postnatal epression scale (EPDS): Translated versions - validated. Perth: State Perinatal mental Health Reference Group; 2006.

21. Brislin RW. Back-translation for cross-cultural research. J Cross-Cultural Psychol. 1970;1(3):185-216.

22. Hanlon C, Medhin G, Alem A, Araya M, Abdulahi A, Tesfaye M, Wondimagegn D,Taha $\mathrm{H}$, Anbesse B, Baheretibeb Y. Measuring common mental disorders in women in Ethiopia. Soc Psychiatry Psychiatr Epidemiol. 2008:43(8):653-9.

23. Fisher J, Morrow M, Nhu NN, Hoang AL. Prevalence, nature, severity and correlates of postpartum depressive symptoms in Vietnam. Int J Obstetr Gynaecol. 2004;111(12):1353-60.

24. Nepal MK, Sharma VD, Koirala NR, Regmi SK, Khalid A, Shresta P. Validation of the Nepalese version of Edinburg Postnatal Depression Scale in the tertiary health care facilities in Nepal. Nepalese J Psychiatry. 1999;1:34-40.

25. Gausia K, Fisher C, Algin S, Oosthuizen J. Validation of the Bangla version of the Edinburgh Postnatal Depression Scale for a Bangladeshi sample. J Reprod Infant Psychol. 2007;25(4):308-15.

26. Husain N, Kiran T, Sumra A, Zafar SN, Rahman RU, Jafri F, Ansari S, Husain M, Adelekan ML, Chaudhry IB. Detecting maternal depression in a low-income 
country: Comparison of the Self-Reporting Questionnaire and the Edinburgh Postnatal Depression Scale. J Trop Pediatr. 2013;60(2):129-33.

27. Tran TD, Tran T, La B, Lee D, Rosenthal D, Fisher J. Screening for perinatal common mental disorders in women in the north of Vietnam: A comparison of three psychometric instruments. J Affect Disord. 2011;133(1):281-93.

28. Patel V, Rodrigues M, DeSouza N. Gender, poverty, and postnatal depression: A study of mothers in Goa, India. Am J Psychiatry. 2002;159(1):43-7.

29. Fernandes M, Srinivasan C, Krishnamachari S, Stein A, Menezes G, Sumithra $R$, Ramchandani P. Assessing prenatal depression in the rural developing world: A comparison of two screening measures. Arch Womens Ment Health. 2011;14(3):209-16.

30. Uwakwe R. Affective (depressive) morbidity in puerperal Nigerian women: Validation of the Edinburgh Postnatal Depression Scale. Acta Psychiatr Scand. 2003;107(4):251-9.

31. Adewuya AO, Ola BA, Dada AO, Fasoto OO. Validation of the Edinburgh Postnatal Depression Scale as a screening tool for depression in late pregnancy among Nigerian women. J Psychosom Obstetr Gynecol. 2006;27(4):267-72.

32. Pollock Jl, Manaseki-Holland S, Patel V. Detection of depression in women of child-bearing age in non-western cultures: A comparison of the Edinburgh Postnatal Depression Scale and the Self-Reporting Questionnaire-20 in Mongolia. J Affect Disord. 2006;92(2-3):267-71.

33. Hanlon C, Medhin G, Alem A, Araya M, Abdulahi A, Hughes M, Tesfaye M, Wondimagegn D, Patel V, Prince M. Detecting perinatal common mental disorders in Ethiopia: validation of the self-reporting questionnaire and Edinburgh Postnatal Depression Scale. J Affect Disord. 2008;108(3):251-62.

34. Stewart RC, Umar E, Tomenson B, Creed F. Validation of screening tools for antenatal depression in Malawi-A comparison of the Edinburgh Postnatal Depression Scale and Self Reporting Questionnaire. J Affect Disord. 2013; 150(3):1041-7.

35. Tesfaye M, Hanlon C, Wondimagegn D, Alem A. Detecting postnatal common mental disorders in Addis Ababa, Ethiopia: Validation of the Edinburgh postnatal depression scale and kessler scales. J Affect Disord. 2010;122(1):102-8.

36. Chibanda D, Mangezi W, Tshimanga M, Woelk G, Rusakaniko P, Stranix-Chibanda L, Midzi S, Maldonado Y, Shetty AK. Validation of the Edinburgh Postnatal Depression Scale among women in a high HIV prevalence area in urban Zimbabwe. Arch Womens Ment Health. 2010;13(3):201-6.

37. Rowel D, Jayawardena P, Fernando N. Validation of the Sinhala translation of Edinburgh Postnatal Depression Scale. Ceylon Med J. 2008:53(1):10-3.

38. Weobong B, Akpalu B, Doku V, Owusu-Agyei S, Hurt L, Kirkwood B, Prince $M$. The comparative validity of screening scales for postnatal common mental disorder in Kintampo, Ghana. J Affect Disord. 2009;113(1):109-17.

39. Rahman A, lqbal Z, Lovel H, Shah MA. Screening for Postnatal depression in the Developing world: A comparision of the WHO self reporting questionnaire (SRQ) and the Edinburg postnatal depression screen (EPDS). J Pakistan Psychiatr Soc. 2005;2(2):69.

40. Regmi S, Sligl W, Carter D, Grut W, Seear M. A controlled study of postpartum depression among Nepalese women: Validation of the Edinburgh Postpartum Depression Scale in Kathmandu. Trop Med Int Health. 2002;7(4):378-82.

41. Glover TA, Albers CA. Considerations for evaluating universal screening assessments. J Sch Psychol. 2007;45(2):117-35.

42. High level International round table on literacy "Reaching the 2015 Literacy Target: Delivering on the promise" UNESCO, Paris, 6-7 September 2012: Action Plan

43. Small R. An Australian Study of Vietnamese, Turkish and Filipino Women's Experiences of Maternity Care and of Maternal Depression After Childbirth [Doctor of Philosophy]. Melbourne: La Trobe University, 2000.

\section{Submit your next manuscript to BioMed Central and we will help you at every step:}

- We accept pre-submission inquiries

- Our selector tool helps you to find the most relevant journal

- We provide round the clock customer support

- Convenient online submission

- Thorough peer review

- Inclusion in PubMed and all major indexing services

- Maximum visibility for your research

Submit your manuscript at www.biomedcentral.com/submit

) Biomed Central 